\title{
Life Course Influences on Inequalities in Later Life: Comparative Perspectives
}

\author{
Hal Kendig ${ }^{1}$ - James Nazroo ${ }^{2}$
}

Published online: 28 December 2015

(C) Springer Science+Business Media Dordrecht 2015

The papers contained in this special issue of the Journal of Population Ageing originated in a session held at the 2014 International Sociological Association Conference, for which we organised a symposium for the Research Committee on Sociology of Aging, sharing the same title as this editorial. The main motivation for the symposium, and for this issue, is the recognition that outcomes in later life are influenced by social advantages and disadvantages over the life course. Of course this is a truism, but understanding the mechanisms through which such life course influences operate, and how context shapes them, is crucial to informing policy aimed at improving outcomes in later life (Dannefer and Settersten 2010). For example, we might consider how investments in childhood and early adulthood (such as in education, housing and welfare) set trajectories of accumulating advantage and disadvantage to influence outcomes in later life, or how negative trajectories into later life that might be common for some population groups can be 'broken' by such policy driven investments.

The importance of policy development with later life in mind cannot be overstated in the context of population ageing. This will not be a new argument for the readers of this journal, but it is worth recognising that much of the research that we undertake and publish makes only passing reference to the importance of the life course, even when there is a focus on inequalities. Policy responses require a thorough understanding of the most effective points in the life course for building resources and interventions that yield ongoing benefits for individuals and societies. The life course perspective provides a valuable corrective to crosssectional descriptions and policy discussions that focus on differences between age groups and generations, often in ideological laden debates on generational advantage and intergenerational justice without an acknowledgement of inequalities within generations and the importance of structural drivers of inequalities within and across generations. For adequate policy development it is crucial to elucidate the underlying social processes that underlie the emergence of inequalities over the life span.

James Nazroo

james.nazroo@manchester.ac.uk

1 Centre for Research on Ageing, Health, and Well-being, Research School of Population Health, Australian National University, Canberra, Australia

2 Sociology and MICRA, School of Social Sciences, University of Manchester, Manchester, UK 
In terms of studying such life course mechanisms there is much that can be learnt from the conceptual and methodological tools of life course epidemiology (Kuh and Ben-Shlomo 2004, where various models have been offered. The most influential of these is the critical period model, where it is proposed that influences occurring during a particular, sensitive, phase of life have implications on outcomes later in life. Often this is presented in relatively narrow terms, with the focus usually on early childhood or prenatal development and the assumption that exposures at this stage will have irreversible consequences that directly determine outcomes and development throughout life. This, of course, downplays other mechanisms that operate later in, or throughout, the life course, and the possibility that early life effects might be aggravated, or mitigated, by events and circumstances later in life. Importantly, such a narrow focus runs the risk of encouraging policy to focus exclusively on early life, as reflected, for example, in the Marmot led reviews of inequalities in health (Marmot 2010) and to ignore the possibility of influences and interventions that make meaningful contributions at other stages in the life course, including in later life.

Unlike the critical period model, the accumulation model considers, as its name implies, the way in which cumulative insults or exposures during the life course impact on later life outcomes, without much attention placed on the timing of these events. For example, we might hypothesise that the longer the duration of unemployment over the life course, regardless of when it occurred, the more adverse the impact on later life health, employment, material circumstances, social participation, quality of life, and happiness. Important here is that alongside the accumulation of risk this model implies that inequalities widen throughout the life course - those with the most advantage become more advantaged over time, and those with the most disadvantage become more disadvantaged over time. This is something that Dannefer (2003) has described as a cumulative advantage/disadvantage model and one that raises 'questions of fairness in the distribution of opportunities and resources' (Dannefer 2003, p.S327), the opportunities and resources that result in accumulation. Centrally important here is to consider the possibility of social mobility and how this might mitigate the accumulation of risk factors.

Related to the possibility that inequalities within a cohort increase over the life course, is the importance of paying attention to the pathways through which events and circumstances at one point in the life course might indirectly influence those at a later point. What might be called a pathways model. Here it is important to pay attention to factors that might mediate the relationship between circumstances earlier and later in the life course, and how such mediating factors might be influenced (Pavalko and Caputo 2013).

Underlying all three of these broad mechanisms is the need to take account of trajectories across the life course, as Elder (1998) has recommended. This involves not only examining the connections between events and circumstances at different stages of the life course, but also paying attention to how these connections might be framed as trajectories, and diverse trajectories, across the life course, how trajectories are set, and how movement onto disadvantageous trajectories might occur and movement from disadvantageous trajectories might be achieved.

The life course framework, and the models underlying it, provides the opportunity to shed important light on how the interplay between individual life experiences and social structures impact on outcomes in later life. The life course is influenced by a 
range of social, historical, environmental and cultural processes. However, the multiple trajectories and social pathways taken by individuals through the life course are strongly influenced by historical and national (and sub-national) context - variations in demography, institutions and cultural/social expectations across time and place will lead to important differences in meanings, influences and opportunities in relation to the life course. Here, the intersections between cohorts and periods is a crucial consideration, the possibility that differences in circumstances for one cohort compared with another (such as retirement opportunities) will lead to different types of life course trajectories, or that differences in circumstances for one period compared to another period (such as a recession) will lead to differences in important influences across several cohorts.

A classic example of these issues can be found in discussions of the baby boomer cohort in the context of population ageing, stagnating real incomes, and the retrenchment of the welfare state. For the baby boomer cohort a combination of demographic, economic, technological, education and welfare changes are said to have led to unprecedented opportunities for cultural changes. The opportunities for and life courses of baby boomers are argued to be unlike those of any preceding generations and may be greater than for generations that are following them (Leach et al. 2013). Alongside this, of course, the institutional and economic changes experienced by baby boomers also impacted on earlier and later cohorts, and the cultural innovations they were central to were also transmitted to and were participated in by earlier and later cohorts. A valuable consideration here is to consider how at a particular point of time there are interdependencies between the experiences of different cohorts - a so-called linked lives perspective that can be considered in relation to intimately linked individuals and families that extend across lineages and cohorts (for example, how childcare policies will impact on each of children, parents and grandparents) and that can be considered in relation to broad cohorts (for example, how welfare spend is split across child benefit, unemployment welfare, and state pensions, or how taxation policy is fundamental to the intergenerational transmission of wealth and inequalities).

When considering cohort differences and period effects it is also important to consider how they might be differentially experienced across population subgroups, especially when there is a focus on inequalities. For example, how cohort differences in educational opportunities and contraception might have impacted on men and women differently, or how period differences in employment opportunities may have a differential impact on men and women, or those from different ethnic groups, or different class groups.

Perhaps not obvious here is the value of also considering cross-national differences in demographic, cultural and institutional structures that influence life courses and change at different paces, and perhaps in different directions, over time (Niedzwiedz et al. 2014). Cross-national comparisons can, therefore, exploit such differences to more clearly identify structural influences and policy variations that are not as readily apparent in studies of individuals within one set of social structures. One example of the potential in such a comparison is that between Australia and England. England was more severely affected by WWII and its economy, housing and standards of living recovered far more slowly than in Australia. While both countries had a post-war baby boom, substantial numbers migrated from England (many to Australia), while post-war immigration occurred later to the UK than to Australia and was concentrated around 
Commonwealth countries and driven by different economic imperatives. The UK introduced national health care coverage far earlier than in Australia. Over more recent years, economic indicators suggest that the recent economic crisis impacted earlier and had more profound effects in the UK than Australia. An investigation of the influence of such differences on the life course has the potential to provide powerful, policy relevant, insights.

Having laid out the rationale for examining life course influences on later life outcomes, the mechanisms through which they might operate, and how what might be called a comparative approach (across cohorts, periods, dimensions of social identity, and national contexts) could shed light on influences and mechanisms, it is now worth considering methods and data sources to address these issues. The classic approach to studying the life course is to use prospectively collected longitudinal data from birth cohort and panel studies. Birth cohort studies offer data that has been prospectively collected throughout the life course, including, potentially, observations of pre-natal and even pre-conception circumstances. They have the disadvantage of covering only one cohort, typically in one national context, but comparisons can be drawn across different birth cohort studies. And also carry the disadvantage of data on later life not being available until a very long time after the study commences. Nevertheless, comparisons across cohorts would allow predictions on how currently young cohorts might age and how this might inform policy development.

Longitudinal data from panel studies carry the advantage of containing several cohorts within the same study that, after a sufficient period of observation, allows for a direct comparison of different cohorts as they grow older. However, they typically rely on retrospectively collected data to cover early life and, for the panel studies of ageing, also mid life. Although the use of calendar (life grid) techniques greatly improve the quality of such data (Belli et al. 2007, Berney and Blane 1997), they nevertheless run the risk of bias and some forms of data, such as those on attitudes, cannot be collect retrospectively. And, the composition of older parts of the samples of such panel studies will be heavily influenced by survival effects, whereby the most vulnerable will have died and, consequently, will not be observed meaning that important life course influences for them may well be missed. It is also worth highlighting the value of qualitative data. Qualitative approaches allow for the collection of high quality biographical data, perhaps using creative methods (such as photo, or object, eliCitation), whose completeness and accuracy can be interrogated during the data collection process, and where personal and social meanings of events and circumstances can be explored and constraints, choices and responses in relation to them understood.

The papers in this volume reflect some of the range of research opportunities described above, each shedding light on the value of different aspect of the life course approach. While they all make empirical contributions, the papers vary in terms of the countries studied, the disciplines of the authors and their focus on outcomes, pathways, life stage, cohorts, and methods. Worth highlighting is that many of the papers move beyond the traditional epidemiological focus on health outcomes to examine later life wellbeing. Research based on the views of older people themselves demonstrates how they value wellbeing outcomes that centre around continuity of identity and established ways of life as guides to informing health promotion on their behalf (Kendig et al. 2014). Significant advances in theorising and measuring wellbeing in later life provides 
novel opportunities to provide a more comprehensive understanding of the drivers of inequality in later life (Vanhoutte 2014), an opportunity that has been taken by papers in this volume.

In the first paper, 'Work and Family Trajectories: Changes Across Cohorts Born in the First Half of the 20th Century', Scherger and colleagues take advantage of the retrospectively collected life history data contained in the English Longitudinal Study of Ageing (ELSA), collected using calendar methods. They use these data to examine cohort differences in family formation and employment histories, and, in particular, to examine whether the baby boomer generation was path breaking in terms of life course trajectories. To examine this possibility they use sequence and cluster analyses to identify trajectories within each cohort and examine associations with gender and class. They conclude that the empirical evidence does not support more theoretically based claims that a single cohort, baby boomers, was particularly pioneering.

Also examining work and family trajectories, Lacey and colleagues use prospectively collected birth cohort data from the MRC National Survey of Health and Development (the 1946 British birth cohort study, who could be considered to be early baby boomers) to explore how these trajectories impact on later life wellbeing. They identify trajectories across the ages 16 to 60 , again using sequence analysis, to predict wellbeing at age 60-64 and show how wellbeing relates to ties to work and family, and the combination of the two, and how this differs for men and women. They go on to highlight the need for policy to pay attention to how work and family life are combined.

Kendig and colleagues also examine the baby boomer cohort in their paper 'Pathways to Well-Being in Later Life: Socioeconomic and Health Determinants Across the Life Course of Australian Baby Boomers'. Here, though, they examine a different national context and focus on socioeconomic inequalities within this specific cohort. Again they use retrospectively collected life history data, using calendar methods. However, rather than using sequence analysis to examine trajectories, they use path analysis to investigate direct and indirect effects of childhood and adult socioeconomic status and health on wellbeing in later life. Their findings support an accumulation of disadvantage model, but are also consistent with a critical period and pathways model, with childhood exposures having small but significant direct (critical period) and indirect (pathways) effects. They argue that policy should focus on the importance of circumstances and events occurring during adulthood as well as those during childhood.

Another paper taking advantage of the robust collection of detailed retrospective life history data to examine the influence of working life on later life circumstances is that by Wahrendorf and colleagues 'Linking Quality of Work in Midlife to Volunteering During Retirement: a European Study'. This study pools the data from the 13 European countries contained in the Survey of Health, Ageing and Retirement in Europe (SHARE) and shows the long-term negative influences of stressful work during adulthood on participation in voluntary work in older ages, and that this relationship is robust to a variety of statistical controls. They conclude that promoting good working conditions may not only increase health and wellbeing, but also encourage participation in productive activities after labour market exit.

Vanhoutte and Nazroo move beyond a single nation or pooled nation study to comparatively examine the importance of life course socioeconomic conditions across two nations in their paper 'Life Course Pathways to Later Life Wellbeing: A 
Comparative Study of the Role of Socio-Economic Position in England and the U.S.'. Here they use the comparable data produced by the Health and Retirement Survey and the English Longitudinal Study of Ageing to examine life course influences on wellbeing in later life, providing strong evidence for the importance of the accumulation of advantage and disadvantage across the life course, and for the role of social mobility, with more limited evidence for a critical period/early life effect. Importantly, they show that both early life and life course effects have a larger influence in the U.S. than in England, suggesting important national differences in the extent of accumulation across the life course.

The last two papers in this special issue focus more specifically on retirement. Hokema and Scherger take advantage of a cross-country comparison (Germany and the UK) to examine system effects (gendered welfare regimes) on gender and marital status differences in the presence of and motivation for working post state pension age. This is the only paper in this special issue that utilises qualitative methods, which it does alongside quantitative methods to show how the findings revealed from the analysis of the quantitative data might be explained. In this way the authors show how financial motivations for continuing to work are much more important for some categories of women compared with other categories of women and men in general, but that the specifics of this varies across the two countries and is strongly related to gender differences in labour market opportunities.

The second of the papers dealing with retirement examines changes in self-reported illness at the retirement transition. For this, Marshall and Nazroo use ten years of prospectively collected data from ELSA, which allows them to model observed trajectories in health prior to and after observed retirement. Rather than using sequence analysis (as done by the other papers modelling trajectories in this special issue), they use repeated measures logistic regression in a spline model and observe that the increase in self-reported illness after retirement is slower than that before retirement. However, they find that this is particularly the case for those in lower social classes, in physically demanding occupations, who were depressed prior to retirement and who were single. They conclude that it is important for policy to consider the demands of work for those in the least advantaged jobs and that this is particularly important when developing policies to increase retirement age, which might otherwise result in a widening of inequalities in later life.

In considering this volume as a whole, it is important to appreciate that they are based on large investments in the tools needed for social science research, most notably the birth cohort studies and the comparable panel studies and longitudinal data sets in Europe and North America (notably ELSA, HRS, and SHARE). This in turn has depended on the far-sighted vision of major funders in supporting systematic, comparable, research across cohorts and across countries (Suzman and Harper 2013). Continuing these data sets while making adjustments for evolving social change will be vital for future research. Having comparable life course data from a broader range of countries, including rapidly developing countries such as China (CHARLS) and India (LASI), can extend knowledge of processes of social change as well as the influence of demographic, cultural, institutional and economic context.

In conclusion, we would argue that the life course approach is opening new research directions that can enable us to link individual lives over the life span with the varying and changing social structures that shape these lives. Comparing the life experiences of 
successive cohorts can shed light on social change while comparisons between countries can shed light on the effects of uneven economic, social, and policy developments. Investigation of varying family, work, and other life trajectories can provide insights into the underlying determinants of health, productivity, social participation, and wellbeing in later life. Taken together these approaches can build a comprehensive knowledge base for understanding ways to address social inequalities for individuals and social groups, as well as to build policy capacity to respond constructively and proactively to the opportunities and challenges of population ageing.

\section{References}

Belli, R. F., Smith, L. M., Andreski, P. M., \& Argrawal, S. (2007). Methodological comparisons between CATI event history calendar and standardized conventional questionnaire instruments. Public Opinion Quarterly, 71(4), 603-622.

Berney, L. R., \& Blane, D. B. (1997). Collecting retrospective data: accuracy of recall after 50 years judged against historical records. Social Science \& Medicine, 45(10), 1519-1525.

Dannefer, D. (2003). Cumulative advantage/disadvantage and the life course: cross-fertilizing age and social science theory. The Journals of Gerontology Series B: Psychological Sciences and Social Sciences, 58(6), S327-S337.

Dannefer, D., \& Settersten, R. (2010). The study of the life course: implications for social gerontology. In D. Dannefer, \& C. Phillipson (Eds.), The SAGE handbook of social gerontology (pp. 4-19). London: Sage Publications.

Elder, G. H. (1998). The life course as developmental theory. Child Development, 69(1), 1-12.

Kendig, H., Browning, C., Thomas, S., \& Wells, Y. (2014). Health, lifestyle, and gender influences on aging well: an Australian longitudinal analysis to guide health promotion. Frontiers in Public Health, 2(70), 19.

Kuh, D., \& Ben-Shlomo, Y. (2004). A life course approach to chronic disease epidemiology. New York: Oxford University Press.

Leach, R., Phillipson, C., Biggs, S., \& Money, A. (2013). Baby boomers, consumption and social change: the bridging generation? International Review of Sociology, 23(1), 104-122.

Marmot, M. (2010). Fair society, healthy lives. The marmot review. Strategic review of health inequalities in England post-2010. London: Institute for Health Equity, University College London.

Niedzwiedz, C. L., Katikireddi, S. V., Pell, J. P., \& Mitchell, R. (2014). The association between life course socioeconomic position and life satisfaction in different welfare states: European comparative study of individuals in early old age. Age and Ageing, 43(3), 431-436.

Pavalko, E. K., \& Caputo, J. (2013). Social inequality and health across the life course. American Behavioral Scientist, 57(8), 1040-1056.

Suzman, R., \& Harper, S. (2013). Editorial: dawning of a new age for longitudinal cohort data. Journal of Population Ageing, 6, 1-4.

Vanhoutte, B. (2014). The multidimensional structure of subjective well-being in later life. Journal of Population Ageing, 7(1), 1-20. 